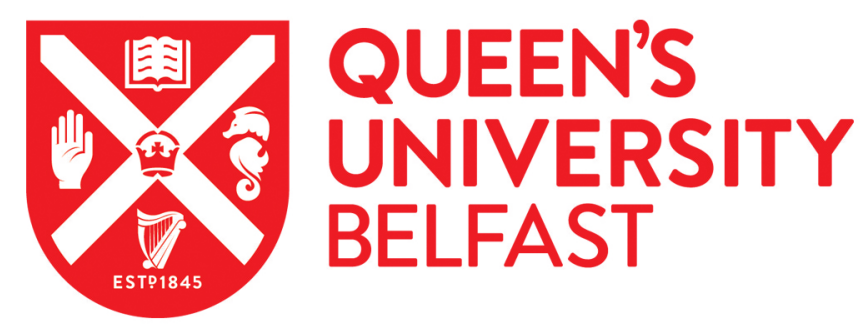

\title{
Atraumatic vs conventional restorative treatment for root caries lesions in older patients: Meta- and trial sequential analysis
}

Göstemeyer, G., DaMata, C., McKenna, G., \& Schwendicke, F. (2019). Atraumatic vs conventional restorative treatment for root caries lesions in older patients: Meta- and trial sequential analysis. Gerodontology. https://doi.org/10.1111/ger.12409

\section{Published in:}

Gerodontology

\section{Document Version:}

Peer reviewed version

Queen's University Belfast - Research Portal:

Link to publication record in Queen's University Belfast Research Portal

Publisher rights

(C) 2019 Gerodontology Association and John Wiley \& Son.

This work is made available online in accordance with the publisher's policies. Please refer to any applicable terms of use of the publisher.

\section{General rights}

Copyright for the publications made accessible via the Queen's University Belfast Research Portal is retained by the author(s) and / or other copyright owners and it is a condition of accessing these publications that users recognise and abide by the legal requirements associated with these rights.

Take down policy

The Research Portal is Queen's institutional repository that provides access to Queen's research output. Every effort has been made to ensure that content in the Research Portal does not infringe any person's rights, or applicable UK laws. If you discover content in the Research Portal that you believe breaches copyright or violates any law, please contact openaccess@qub.ac.uk. 


\section{Atraumatic versus Conventional Restorative Treatment for Root Caries Lesions in Older Patients: Meta- and Trial Sequential Analysis}

Short title: ART for root caries - systematic review

Gerd Göstemeyer ${ }^{{ }^{*}}$, Cristiane da Mata ${ }^{2}$, Gerald McKenna ${ }^{3}$, Falk Schwendicke ${ }^{1}$

${ }^{1}$ Department of Operative and Preventive Dentistry, Charité - Universitätsmedizin Berlin, Germany

${ }^{2}$ Cork University Dental School and Hospital, University College Cork, Ireland

${ }^{3}$ Centre for Public Health, Queens University Belfast, UK

${ }^{*}$ Corresponding author:

Dr. Gerd Göstemeyer

Charité Centre for Dental Medicine

Department for Operative and Preventive Dentistry

Aßmannshauser Str. 4-6

14197 Berlin

Germany

Phone 004930450562328

Fax 004930450562932

gerd.goestemeyer@charite.de 


\section{Abstract}

Objectives: We aimed to appraise the comparative clinical efficacy of atraumatic restorative treatment (ART) versus conventional restorative treatment (CT) using a meta-analysis and assessed the robustness of evidence by trial sequential analysis (TSA).

Background: Due to its simplified clinical approach, ART may be advantageous over CT for restoration of root caries lesions in institutionalized older patients.

Methods: Three electronic databases (PubMed, Embase, Cochrane CENTRAL) were screened and hand searches and cross-referencing performed to identify randomized controlled trials reporting on survival of ART versus CT for restoration of root caries in older patients. Trial selection, data extraction, and risk of bias assessment was performed by two independent reviewers. ART and CT were compared using fixed- or random-effects pairwise meta analysis for per-protocol (PP), intention-to-treat (ITT) and best case scenarios. TSA was used to control for risk of random errors.

Results: 235 studies were identified and 3 trials involving 130 patients (463 restorations) included. Risk of bias was high or moderate in all but one trial. ART was associated with a significantly increased risk of failure (OR $[95 \% \mathrm{Cl}] 2.06[1.06 / 4.00])$ in PP- but not in ITTanalysis (1.36 [0.92/2.02]). Analyses for best case scenarios found great uncertainty introduced by attrition. No firm evidence was reached according to TSA.

Conclusions: For restoration of root caries, there is insufficient data to clearly rule out wether differences between ART and CT exist. Limited available data indicate there might be an increased risk of failure for ART.

Keywords: Atraumatic restorative treatment, root caries, geriatric dentistry, evidence-based dentistry 


\section{Introduction}

Considerable advances in the application of caries preventive measures have led to a decline of cavitated caries lesions in younger population groups in most high-income countries ${ }^{1,2}$. However, in frail and/or functionally dependent older people, oral health frequently remains poor ${ }^{3-7}$, with root caries being an increasingly prevalent condition ${ }^{3}$. Given the demographic transition in these countries and more teeth being retained into old age ${ }^{8,9}$, it can be expected that root caries lesions and their management will be a significant challenge in the future ${ }^{6}$. Further challenges arise in managing the growing numbers of dependent older adults within residential care facilitates who may have limited access to dental care ${ }^{10}$ and limited capacity due to cognitive decline. Restoration of root caries lesions presents a number of operative challenges including difficulties with moisture control and bonding to dentine as the sole substrate to adhere to. Hence, alternative approaches for the restoration of root caries lesions may be needed ${ }^{10}$.

Atraumatic Restorative Treatment (ART) was first developed as an approach for restoring carious lesions in people in low income countries, where resources and facilities for dental treatment were limited ${ }^{11}$. Application of ART involves removal of carious tissue by use of hand instruments and restoration of the cavity with an adhesive material, usually high-viscosity glass-ionomer cement (HVGIC). A major advantage of this technique is its applicability in nontraditional clinical settings, since the need for complex instruments and materials is significantly reduced compared to conventional treatment $(\mathrm{CT})$ with rotary equipment. Cavity preparation with hand instruments also reduces patients' pain and discomfort, thus local anesthesia can often be avoided. Restoration of root caries lesions using the ART approach may be more applicable than CT for older patients in different settings such as care facilities, at home or in dental practices.

Systematic reviews are needed to compile the accrued evidence, assess heterogeneity and robustness of the data and deduce meaningful recommendations for clinical decision making. However, evidence on the comparative effectiveness of ART versus other restorative approaches is inconsistent: A recent Cochrane review compared the effects of ART versus CT 
for managing caries in children and adults ${ }^{12}$. It was reported that restorations placed with ART using HVGIC was associated with an increased risk of failure. This was supported by a further systematic review, where GIC restorations had a higher annual failure rate if placed using ART compared to $\mathrm{CT}^{13}$ in load bearing cavities. However, other systematic reviews did not confirm a significant difference in clinical success between amalgam and ART restorations ${ }^{14,15}$. Neither of these reviews compared the comparative efficacy of ART versus CT for restoring root caries lesions. Mechanical forces as well as the dental substrate any restorative material is bonded to differs between root and load-bearing cavity restorations. Therefore, results from available systematic reviews are not generalizable to restorations of root caries. Other review data on ART versus CT restorations for root caries has not been appraised systematically but narratively, focusing only on xerostomic head and neck radiation patients ${ }^{16}$, or posterior cervical lesions in school children which may have limited generalizability when applied to root caries lesions in older adults ${ }^{17,18}$

The aim of this systematic review was to compare the efficacy of ART versus CT for restoring root caries lesions in older adults. To assess the robustness of the synthesized evidence, a Trial Sequential Analysis (TSA) was performed.

\section{Methods}

The reporting of this study is in accordance with the PRISMA and ENTREQ statement guidelines ${ }^{19,20}$.

\section{Eligibility criteria}

This study included Randomized Controlled Trials (RCTs) comparing the survival of ART and CT restorations placed for treatment of root caries lesions in older adults. The following PICO criteria for inclusion of trials were applied:

\section{Population}

Older adults (> 60 years) with root caries lesions in need of restorative treatment were included. Studies were excluded if they only included patients with extreme oral conditions (e.g. studies on patients with head and neck radiation therapy following cancer treatment), to increase the generalizability of our findings. 


\section{Intervention}

Restoration of root caries lesions placed using ART, i.e. cavity preparation using hand instruments only. No restrictions were applied to the restorative materials used.

\section{Control}

Restorations of root caries placed using CT, i.e. cavity preparation using rotary burs. No restrictions were applied to the restorative materials used.

\section{Outcomes}

The outcome studied was restoration failure; possible reasons for failure included retention loss, fracture, caries adjacent to the restoration, or other clinically and/or radiographically determined reasons for replacing or repairing the restoration, as determined by the trial investigators. Note that this broad definition was chosen, as the exact reason for failure may not always be reported, or that multiple reasons may apply. Also note that, eventually, in the included studies, reasons for failure had all been reported in detail, and none fell into this broad category.

\section{Studies}

This systematic review only included RCTs.

\section{Information Sources}

\section{Electronic searches}

Three electronic databases (EMBASE via Ovid, MEDLINE via PubMed, CENTRAL) were screened on 12/08/17 using a defined search protocol, which was adapted for each database. This search was updated on $07 / 11 / 18$ to assess if further trials had been conducted and published. Our search was focused on published articles of original studies. We did not include grey literature such as conference abstracts, books etc. No restrictions were applied to publication language or date. 
Search strategy

A two-pronged search strategy was developed for Medline: (((root) AND caries)) AND (((ART) OR atraumatic) OR restor*) (Filter: Clinical Trial). This strategy was adapted for the other screened databases.

\section{Selection process}

Titles and abstracts of identified studies were independently screened for eligibility by two calibrated reviewers (GG and FS). The reviewers were calibrated according to the criteria for in- and exclusion of trials. Calibration was tested with two papers in a discussion. Inclusion of studies was decided by both reviewers and consensus was obtained by discussion if necessary. Cross-referencing was performed via bibliographies of assessed full-texts and other reviews with a similar scope. There was no disagreement for inclusion of studies.

\section{Data extraction}

Data management

Two reviewers (GG and CdM) independently performed duplicate data extraction, using a piloted spreadsheet.

\section{Data items}

The following data items were extracted:

- Study name, year of publication, location and setting

- Participants (number, gender, age)

- Characteristics of included root caries lesions

- Treatment procedures for Intervention (ART) and Control (CT) including details on cavity preparation/caries removal, moisture control, cavity pretreatment, restorative materials, finishing technique

- Follow-up time, attrition rate, outcome measure, findings

If restoration status had been recorded over several time-points within the same trial, data from the most recent reported time-point was included in this analysis. 


\section{Data synthesis}

Meta-analysis

Meta-analysis was performed for the outcome (risk of failure, measured as events per total restorations in each group) using Review Manager (RevMan) Version 5.3. (The Cochrane Collaboration, 2014). Heterogeneity was assessed using both Cochran's $Q$ and ${ }^{2}$-statistics ${ }^{21}$. Fixed or random-effects meta-analysis was performed depending on heterogeneity $\left(\mathrm{I}^{2}<35 \%\right.$ or above). Odds ratios and $95 \%$ confidence intervals (95\% Cl) were calculated. Publication bias was assessed graphically via a simple test of asymmetry using funnel plots ${ }^{22}$.

To assess the possible impact of attrition on our estimates, meta-analysis was performed for four different scenarios: (a) per-protocol analysis (PP), i.e. assessment of participants based on the intervention they received and that had been followed; participants that dropped-out during follow-up were excluded from analysis. PP accounts for possible attrition bias and deviations from protocol ${ }^{23}$. (b) intention-to-treat analysis (ITT), i.e. assessment of participants as randomized regardless of whether they received the intervention or dropped out. For ITT, we assumed that all missing participants experienced an event (i.e. restoration failure). (c) and (d) scenario analyses following the ITT principle. Here, attrition was handled differently in the experimental and control groups. In the best-case (for ART) analysis (c), we assumed that only drop-outs in the control (CT), but not the experimental (ART) group experienced events (failures). In the best-case (for CT) scenario (d), this was reversed. Scenario analyses explore the uncertainty introduced by attrition via the most extreme imputations.

Trial sequential analysis (TSA)

In a meta-analysis, Z-values are used to compare two interventions. A Z-value of 0 indicates no difference between two interventions. A Z-value exceeding \pm 1.96 corresponds to a $p$-value of $<0.05$ (two-sided test), which is traditionally assumed to indicate a statistically significant difference. For repeated updates of meta-analyses, as new trials become available to include, a new Z-value is calculated for each update. In TSA, this series of Z-values is plotted against the accumulated sample size, events, or information ${ }^{24,25}$. This 'cumulative Z-curve' is then assessed regarding its relation to the conventional significance boundaries $(Z= \pm 1.96)$, the 
required information size (RIS), and the trial sequential monitoring boundaries (TSMB) for benefit, harm, or futility.

Trial Sequential Analysis Viewer 0.9.5.10 Beta (Copenhagen Trial Unit, Centre for Clinical Intervention Research, 2016) was used for conducting the TSA. The RIS was calculated based on an assumed type I error risk of $\alpha=0.05$, a type II error risk of $\beta=0.20$ (equivalent to a power of 0.80 ) and the control event proportion. The relative risk reduction was based on an a priori defined worthwhile interventional effect of $20 \%$. It should be noted that smaller intervention effects may well be relevant ${ }^{26}$. This, however, would increase the RIS even further ${ }^{24}$. Variance based heterogeneity correction was performed according to the O'Brien-Fleming function ${ }^{27}$ and was used for calculating the TSMBs. Results of the cumulative Z-value crossing the conventional boundary of significance $(Z= \pm 1.96)$ but not the TSMBs for benefit or harm were defined as spuriously significant. Firm evidence was assumed to be reached when the Z-curve crossed the outer TSMBs for benefit or harm before the RIS was reached. Firm evidence of futility was confirmed when the Z-curve was crossing the inner TSMBs for futility and the RIS was reached ${ }^{25}$.

\section{Qualitative assessment and confidence in data}

Risk of bias was independently assessed using the Cochrane risk of bias assessment tool ${ }^{28}$ by two reviewers (GG and CdM). The strength of evidence was graded according to the GRADE working group of evidence using the GRADEpro GDT online tool (https://gdt.guidelinedevelopment.org). It should be noted that only the evidence for the PP scenario (a) was graded.

\section{Results}

\section{Results of the searches}

From 332 identified studies, 11 articles were evaluated as full-text manuscripts, and 3 RCTs were included (Fig. 1). Excluded studies and reasons for exclusion can be found in the appendix (Tab. S1). Within the included studies, 463 restorations were placed in 130 patients and assessed with a follow-up time of 6 to 24 month (Tab. 1). 
The age range of participants included in one RCT was 60-101 years and 65-90 years in a second RCT. The other included trial did not report on age range but only the mean age of participants, which was 78.6 years. Two trials were conducted in nursing homes and one trial in a geriatric day hospital and a dental university hospital. In two trials solely root caries lesions were included and in one trial, restorations were placed in both root and coronal caries lesions (the results for the coronal lesions were not included into this review).

All included trials used GIC, HVGIC or resin-modified GIC (RMGIC) as restorative materials. In two trials polyacrylic acid conditioning was performed prior to placing the restoration. In one trial the cavity was conditioned using chlorhexidine instead. Three trials used ART restoration criteria $^{29}$ for evaluation of restorations (one of them additionally used the United States Public Health Service (USPHS)-criteria; one trial used criteria defined by the authors which were build on the ART restoration criteria. The main reasons reported for failure were marginal defects and retention loss.

\section{Meta-analysis}

Fixed-effects meta-analyses were performed for the PP-, ITT- and best case (for CT) scenarios as described in the methods section. Due to significant heterogeneity $\left(I^{2}>35 \%\right)$, randomeffects meta-analyses were performed for the best case (for ART) scenario (Fig. 2). In the PP scenario, ART restorations had a significantly increased risk of failure compared with CT (OR 2.06 [1.06/4.00]) (Fig. 2a). In the ITT scenario, there were no significant differences calculated in risk of failure between ART and CT (OR 1.36 [0.92/2.02]) (Fig. 2b). In extreme scenario analyses, ART had either a significantly decreased risk of failure (OR 0.27 [0.09/0.85]) or an increased (OR 8.39 [4.70/14.87]) risk of failure (Fig. 2 c, d), indicating great uncertainty introduced by attrition. There was no publication bias detected by funnel plot analyses (Appendix Fig. S1).

\section{Trial-sequential analysis}

There was no firm evidence on ART versus CT reached regardless if PP (RIS: 931, reached IS: 353) (Fig 2a) or ITT-scenario analyses (RIS: 1545, reached IS: 463) (Fig 2b) were performed. Neither the TSMB nor RIS boundaries were crossed by the Z-curve. This was also 
found for the best case (for ART) scenario (RIS: 936, reached IS: 463) (Fig. 2c). For the best case (for CT) scenario, firm evidence was reached in favor of CT; the TSMB for benefit of CT was crossed before the Z-curve crossed the RIS.

\section{Risk of bias assessment and evidence grading}

The risk of bias of included trials is shown in Table 2. As blinding of operators and participants could not have been performed in the RCTs, these domains were excluded in the assessment of risk of bias. We found low risk of bias in one trial, moderate risk of bias in another trials and high risk of bias in a further trial. The GRADE summary table revealed that the certainty of the evidence from our findings was low (Appendix Fig S2).

\section{Discussion}

Due to its reduced instrumental and technical requirements ART can be provided outside of the traditional clinical environment, with less pain and discomfort for the patient. As the technique is minimally invasive, ART may theoretically be associated with a lower risk of pulp exposure and the subsequent need for further complex operative treatments. Given these potential advantages, ART may be more applicable for managing root caries in dependent older adults, particularly those in residential care or as part of domiciliary care. Based on limited data this systematic review and meta-analysis indicates that ART may be associated with an increased risk of restoration failure compared to CT. Our TSA revealed that firm evidence was not reached to draw a clear conclusion. High attrition rates in the included trials further weakened the validity of our findings.

For PP analysis, we found ART was associated with a significantly increased risk of failure compared to CT. Our finding is in line with other systematic reviews where ART also tended to perform worse than CT in various situations ${ }^{12,13,17}$. Other systematic reviews could not demonstrate a difference between ART placed with HVGIC and amalgam restorations ${ }^{14,15}$. Given this uncertainty, more high quality randomized-controlled trials are recommended to 
assess the comparative clinical effectiveness of ART restorations not only for root caries, but for various indications.

Higher failure rates of ART might be the result of less controlled conditions in terms of contamination control and cavity preparation when placing the restoration ${ }^{13}$. However, in trials included into our review, the conditions in which the restoration were placed were not reported to differ between ART and CT in terms of contamination control. Higher rates of partly or completely loss of restorative material in ART restorations might also be the result of a reduced adhesion of the restoration to caries affected areas of the cavities prepared according to ART principles ${ }^{30}$. This adhesion may be of importance especially in root caries, where retentive cavity preparation is not always possible (considering the often saucer-shaped cavities).

Another main reasons for failure of ART root caries restorations were marginal defects. The press finger technique and the lack of polishing, as part of the ART procedure, may result in rougher restoration surfaces and margins, which can act as plaque retention areas ${ }^{30}$. Given the highly cariogenic conditions in frail older people, eliminating such areas may be important for the clinical success of restorations. Further trials should assess whether greater efforts in furbishing ART restorations can improve their survival.

Based on TSA, firm evidence of our findings was not reached for any scenario (except the best-case (for CT) scenario, which represents an extreme situation unlikely to have occurred). Hence, TSA indicates great uncertainty of findings: even for the more realistic PP-scenario, where we found a significantly increased risk of failure for ART restorations according to the meta-analysis, TSA revealed that this result might be spuriously significant. However, TSA calculated a RIS of 931 of treated lesions to reach firm evidence for this scenario. Given that 423 lesions from the RIS have already been treated in the 4 included trials, the number of lesions needed to treat to reach firm evidence seems manageable. It should be noted that we did not account for clustering in our analyses, as the unit of calculation in our study was the 
lesion and not the patient. Accounting for such clustering may further widen the confidence intervals, i.e. aggravate uncertainty.

Our review has further limitations. First, only three RCTs were included. These studies demonstrated some heterogeneity in terms of clinical technique and the clinical environment where the treatment took place. In particular, different types of GICs were used for the placement of ART restorations. It is clear that the type of restorative material may impact restoration survival. However, ART restorations performed worse compared to CT (which were all placed using RMGIC) throughout all included trials irrespective of the GIC type used. This indicates that the ART approach might be generally associated with an increased risk of failure in root caries restorations irrespective of which restorative material has been used. One of the included trials used $\mathrm{CHX}$ as cavity pretreatment. This treatment step is usually applied before placing resin-based restorations. However, its a clinical effect on adhesively placed restorations is still unclear ${ }^{31}$. For GIC, no clinical data is available supporting or prohibiting its use prior placement of a restoration. We further did not set a maximum age of participants as exclusion criteria to potentially improve a homogeneous distribution of risk factors between patients. Given that all trials were predominantly performed in almost similar geriatric settings and all trials were randomized we assumed that such risk factors were balanced within and across trials. The included trials had different follow-up periods ranging from 6 to 24 month. Pooling these data allows to draw conclusions about the relative effect of the two interventions in general. However, for reviews with a high number of studies, subgroup analyses according to follow-up period may be performed, while in reviews with only limited included studies (as ours), such subgrouping is usually not particularly useful and even prone for erroneous conclusions due to lacking power. Second, all included studies reported large attrition rates, which is also expectable given the age of the study population and their life expectancy. We explored the relevance of this attrition by performing scenario analyses and found that depending on the assumed scenario, everything (from benefits of CT to possible benefits of ART) seems possible. Further trials should account for the expectedly high attrition rates in 
the target population in their power calculation. Third, the failure rates of both ART and CT restorations was high, which illustrates the challenges of restoring root caries lesions in institutionalized older patients ${ }^{32}$ and calls into question if restorative approaches in these situations are generally an appropriate therapy. Alternative non-invasive treatment options (e.g. application of Silver Diamine Fluoride (SDF)) may be effective for managing root caries lesions without restoring them ${ }^{33,34}$. However, the effectiveness of SDF versus restorations of root lesions has not been assessed yet (as it is still uncommon to compare non-invasive and invasive caries management strategies ${ }^{35}$ ). Fourth, a simple search strategy was used for the searching the electronic databases. However, as we also performed cross-referencing from included RCTs and other systematic reviews, it might be unlikely that relevant publications have been missed. Fifth, this review was not registered. One main goal of registering systematic reviews is to avoid unplanned duplication of reviews. So far, we did not identify reviews with the same direction as ours. Last, assessing restoration failure as a surrogate for the clinical effectiveness of the treatment might be debatable, especially in light of the demands of institutionalized older people. Other parameters might be more important for the quality of life of this population group, and patient-related outcomes should be collected by future studies 36.

\section{Conclusion}

This systematic review and meta-analysis found no compelling evidence to support either ART or $\mathrm{CT}$ for restoring root caries lesions in older adults. However, based on the limited number of included trials, CT may be more efficacious than ART for this purpose. As only a limited number of patients is needed to reach firm conclusions on the efficacy of ART versus CT, future trials seem worthwhile. 


\section{References}

1. Lagerweij MD, van Loveren C. Declining Caries Trends: Are We Satisfied? Current oral health reports. 2015;2(4):212-217.

2. Beikler T, Flemmig TF. Oral biofilm-associated diseases: trends and implications for quality of life, systemic health and expenditures. Periodontology 2000. Feb 2011;55(1):87-103.

3. Hariyani N, Setyowati D, Spencer AJ, Luzzi L, Do LG. Root caries incidence and increment in the population - A systematic review, meta-analysis and meta-regression of longitudinal studies. Journal of dentistry. Oct 2018;77:1-7.

4. Petersen PE, Kandelman D, Arpin S, Ogawa H. Global oral health of older people--call for public health action. Community dental health. Dec 2010;27(4 Suppl 2):257-267.

5. Eke PI, Wei L, Borgnakke WS, et al. Periodontitis prevalence in adults $>/=65$ years of age, in the USA. Periodontology 2000. Oct 2016;72(1):76-95.

6. Lopez R, Smith PC, Gostemeyer G, Schwendicke F. Ageing, dental caries and periodontal diseases. Journal of clinical periodontology. Mar 2017;44 Suppl 18:S145S152.

7. De Visschere LM, Grooten L, Theuniers G, Vanobbergen JN. Oral hygiene of elderly people in long-term care institutions--a cross-sectional study. Gerodontology. Dec 2006;23(4):195-204.

8. Muller F, Naharro M, Carlsson GE. What are the prevalence and incidence of tooth loss in the adult and elderly population in Europe? Clinical oral implants research. Jun 2007;18 Suppl 3:2-14.

9. Schwendicke F, Krois J, Kocher T, Hoffmann T, Micheelis W, Jordan RA. More teeth in more elderly: Periodontal treatment needs in Germany 1997-2030. Journal of clinical periodontology. Oct 92018.

10. Bots-VantSpijker PC, Vanobbergen JN, Schols JM, Schaub RM, Bots CP, de Baat C. Barriers of delivering oral health care to older people experienced by dentists: a systematic literature review. Community dentistry and oral epidemiology. Apr 2014;42(2):113-121.

11. Frencken JE, Pilot $T$, Songpaisan $Y$, Phantumvanit $P$. Atraumatic restorative treatment (ART): rationale, technique, and development. Journal of public health dentistry. 1996;56(3 Spec No):135-140; discussion 161-133.

12. Dorri M, Martinez-Zapata MJ, Walsh T, Marinho VC, Sheiham Deceased A, Zaror C. Atraumatic restorative treatment versus conventional restorative treatment for managing dental caries. The Cochrane database of systematic reviews. Dec 28 2017;12:CD008072.

13. Ruengrungsom C, Palamara JEA, Burrow MF. Comparison of ART and conventional techniques on clinical performance of glass-ionomer cement restorations in load bearing areas of permanent and primary dentitions: A systematic review. Journal of dentistry. Jul 112018.

14. Mickenautsch S, Yengopal V. Failure rate of high-viscosity GIC based ART compared with that of conventional amalgam restorations--evidence from an update of a systematic review. SADJ : journal of the South African Dental Association = tydskrif van die Suid-Afrikaanse Tandheelkundige Vereniging. Aug 2012;67(7):329-331.

15. Mickenautsch S, Yengopal V. Failure Rate of Direct High-Viscosity Glass-lonomer Versus Hybrid Resin Composite Restorations in Posterior Permanent Teeth - a Systematic Review. The open dentistry journal. 2015;9:438-448. 
16. Amer RS, Kolker JL. Restoration of root surface caries in vulnerable elderly patients: a review of the literature. Special care in dentistry : official publication of the American Association of Hospital Dentists, the Academy of Dentistry for the Handicapped, and the American Society for Geriatric Dentistry. May-Jun 2013;33(3):141-149.

17. Mickenautsch S, Yengopal V, Banerjee A. Atraumatic restorative treatment versus amalgam restoration longevity: a systematic review. Clinical oral investigations. Jun 2010;14(3):233-240.

18. Frencken JE, van't Hof MA, Taifour D, Al-Zaher I. Effectiveness of ART and traditional amalgam approach in restoring single-surface cavities in posterior teeth of permanent dentitions in school children after 6.3 years. Community dentistry and oral epidemiology. Jun 2007;35(3):207-214.

19. Tong A, Flemming K, McInnes E, Oliver S, Craig J. Enhancing transparency in reporting the synthesis of qualitative research: ENTREQ. BMC medical research methodology. Nov 27 2012;12:181.

20. Moher D, Hopewell S, Schulz KF, et al. CONSORT 2010 explanation and elaboration: updated guidelines for reporting parallel group randomised trials. Bmj. Mar 23 2010;340:c869.

21. Higgins JP, Thompson SG. Quantifying heterogeneity in a meta-analysis. Statistics in medicine. Jun 15 2002;21(11):1539-1558.

22. Egger M, Davey Smith G, Schneider M, Minder C. Bias in meta-analysis detected by a simple, graphical test. Bmj. Sep 13 1997;315(7109):629-634.

23. Porta N, Bonet C, Cobo E. Discordance between reported intention-to-treat and per protocol analyses. Journal of clinical epidemiology. Jul 2007;60(7):663-669.

24. Keus F, Wetterslev J, Gluud C, Gooszen HG, van Laarhoven CJ. Trial sequential analyses of meta-analyses of complications in laparoscopic vs. small-incision cholecystectomy: more randomized patients are needed. Journal of clinical epidemiology. Mar 2010;63(3):246-256.

25. Wetterslev J, Thorlund K, Brok J, Gluud C. Trial sequential analysis may establish when firm evidence is reached in cumulative meta-analysis. Journal of clinical epidemiology. Jan 2008;61(1):64-75.

26. Thorlund K, Devereaux PJ, Wetterslev J, et al. Can trial sequential monitoring boundaries reduce spurious inferences from meta-analyses? International journal of epidemiology. Feb 2009;38(1):276-286.

27. O'Brien PC, Fleming TR. A multiple testing procedure for clinical trials. Biometrics. Sep 1979;35(3):549-556.

28. Higgins JP, Altman DG, Gotzsche PC, et al. The Cochrane Collaboration's tool for assessing risk of bias in randomised trials. Bmj. Oct 18 2011;343:d5928.

29. Frencken JE, Makoni F, Sithole WD. Atraumatic restorative treatment and glassionomer sealants in a school oral health programme in Zimbabwe: evaluation after 1 year. Caries research. 1996;30(6):428-433.

30. Mickenautsch S, Grossman E. Atraumatic Restorative Treatment (ART): factors affecting success. Journal of applied oral science : revista FOB. 2006;14 Suppl:34-36.

31. Gostemeyer G, Schwendicke F. Inhibition of hybrid layer degradation by cavity pretreatment: Meta- and trial sequential analysis. Journal of dentistry. Jun 2016;49:1421.

32. Hayes $M$, Brady $P$, Burke FM, Allen PF. Failure rates of class $V$ restorations in the management of root caries in adults - a systematic review. Gerodontology. Sep 2016;33(3):299-307. 
33. Zhang W, McGrath C, Lo EC, Li JY. Silver diamine fluoride and education to prevent and arrest root caries among community-dwelling elders. Caries research. 2013;47(4):284290.

34. Tan HP, Lo EC, Dyson JE, Luo Y, Corbet EF. A randomized trial on root caries prevention in elders. Journal of dental research. Oct 2010;89(10):1086-1090.

35. Schwendicke F, Innes N, Levey C, Lamont T, Gostemeyer G. Comparator choice in cariology trials limits conclusions on the comparative effectiveness of caries interventions. Journal of clinical epidemiology. Sep 2017;89:209-217.

36. Levey C, Innes N, Schwendicke F, Lamont T, Gostemeyer G. Outcomes in randomised controlled trials in prevention and management of carious lesions: a systematic review. Trials. Nov 2 2017;18(1):515.

37. Cruz Gonzalez AC, Marin Zuluaga DJ. Clinical outcome of root caries restorations using ART and rotary techniques in institutionalized elders. Brazilian oral research. May 31 2016;30(1).

38. da Mata C, Allen PF, McKenna G, Cronin M, O'Mahony D, Woods N. Two-year survival of ART restorations placed in elderly patients: A randomised controlled clinical trial. Journal of dentistry. Apr 2015;43(4):405-411.

39. Lo EC, Luo Y, Tan HP, Dyson JE, Corbet EF. ART and conventional root restorations in elders after 12 months. Journal of dental research. Oct 2006;85(10):929-932. 